\title{
Acute disseminated encephalomyelitis followed by recurrent or monophasic optic neuritis in pediatric patients
}

Multiple Sclerosis Journal

19(7) 94I-946

(C) The Author(s) 2012

Reprints and permissions:

sagepub.co.uk/journalsPermissions.nav DOI: 10.1 I $77 / 1352458512466317$

msj.sagepub.com

(S)AGE

\section{Peter Huppke', Kevin Rostasy², Michael Karenfort ${ }^{3}$, Brenda Huppke', Rainer Seidl ${ }^{4}$, Steffen Leiz ${ }^{5}$, Markus Reindl ${ }^{6}$ and Jutta Gärtner'}

\begin{abstract}
Background: Some pediatric patients with inflammatory demyelinating central nervous system disorders cannot be classified under any of the established disease entities, making their treatment and prognosis difficult.

Objective: The objective of this study is to characterize a subgroup of pediatric patients with recurrent demyelinating central nervous system disorders.

Methods: This study includes a case series of pediatric patients with monophasic or recurrent acute disseminated encephalomyelitis (ADEM) who later presented with either monophasic or recurrent optic neuritis (ON).

Results: We describe seven patients with a median follow-up of six years (five females, two males) who presented at a median age of 6 years (range 4-8 years) with monophasic $(n=4)$ or recurrent ADEM (two to four attacks) followed by monophasic $(n=3)$ or recurrent ON (two to nine attacks). Cranial magnetic resonance imaging (MRI) was typical for $\operatorname{ADEM}(n=6)$ with complete or almost complete resolution of lesions on follow-up. Cerebrospinal (CSF) studies at the time of ADEM showed a pleocytosis in six patients and were negative for oligoclonal bands (OCBs) in all. In all patients high titers for serum anti-MOG antibodies were detected.

Conclusion: ADEM followed by $\mathrm{ON}$ is a rare but distinct clinical phenotype among pediatric patients. Further studies are needed to allow recommendations on treatment or prognosis.
\end{abstract}

\section{Keywords}

ADEM, NMO, optic neuritis, pediatric

Date received: 9th August 2012; revised: 27th September 20I2; accepted: 9th October 2012

\section{Introduction}

The spectrum of relapsing demyelinating disorders in the pediatric population encompasses multiple sclerosis (MS), representing the majority of patients and less commonly recurrent optic neuritis $(\mathrm{ON})$, recurrent myelitis, neuromyelitis optica (NMO) and recurrent acute disseminated encephalomyelitis (ADEM). ${ }^{1}$ In 2007 consensus definitions for pediatric MS and related disorders were published by the International Pediatric MS Study Group. ${ }^{2}$ Diagnosis of pediatric MS requires, as in adults, evidence of two discrete demyelinating events. However, due to the high frequency of ADEM in this population, it was stated that these events must not meet ADEM criteria. ADEM was defined as "a first clinical event with a presumed inflammatory or demyelinating cause, with acute or subacute onset that affects multifocal areas of the [central nervous system] CNS." The polysymptomatic presentation must include encephalopathy, which was defined as behavioral change or alteration in consciousness, e.g. lethargy. ${ }^{2}$
In 2011 we studied the prevalence of anti-myelinoligodendrocyte glycoprotein (anti-MOG) antibodies in

\footnotetext{
'Department of Pediatrics and Pediatric Neurology, University Medical Center, Georg August University Göttingen, Germany.

2Department of Pediatrics I, Division of Pediatric Neurology, Medical University Innsbruck, Austria.

${ }^{3}$ Department of General Pediatrics and Neonatology, Heinrich-HeineUniversity Düsseldorf, Germany.

${ }^{4}$ Department of Pediatrics, Medical University of Vienna, Austria.

${ }^{5} \mathrm{Hospital}$ for Children and Adolescents, Department of Pediatrics,

Klinikum Dritter Orden München, Germany.

${ }^{6}$ Clinical Department of Neurology, Innsbruck Medical University, Austria

P.H. and K.R. contributed equally to this paper.
}

\section{Corresponding author:}

Peter Huppke, Department of Pediatrics and Pediatric Neurology, Georg August University, Faculty of Medicine, Robert-Koch-Strasse 40, 37075 Göttingen, Germany.

Email: phuppke@med.uni-goettingen.de 
patients with ON. ${ }^{3}$ We became aware of a small subgroup of patients who did not fulfill the diagnostic criteria for any of the relapsing demyelinating disorders described above. These patients presented initially with ADEM or recurrent ADEM and then proceeded with episodes of ON. In this article we give a more detailed description of these patients who were included in the MOG study and further patients not described so far in order to characterize this phenotype of a pediatric inflammatory demyelinating disorder.

\section{Patients and methods}

\section{Data collection}

Data from Patients (Pats) 1-4 were derived from the institutional review board-approved database for inflammatory demyelinating disorders provided by the German Center for Multiple Sclerosis in Childhood and Adolescents at the University Medical Center, Göttingen (Ethic approval number 21/12/03). The database was used previously to describe the clinical characteristics of pediatric MS and NMO.,5 Written informed consent was obtained from all patients (or guardians of patients) participating in the study. The database was searched stepwise for patients presenting with ADEM or recurrent ADEM followed by ON but not fulfilling the diagnostic criteria for pediatric MS. The ON had to occur after a symptom-free interval of four weeks and not as part of the ADEM. In the initial step patients were identified who presented with $\mathrm{ON}$ at least once during the course of their demyelinating disorder. Secondly, all patients fulfilling the criteria for pediatric MS were excluded. ${ }^{2}$ In the final step the remaining patients were reviewed and classified accordingly as NMO, monophasic ON, recurrent ON or ADEM followed by ON. NMO was diagnosed according to the 2005 revised diagnostic criteria: $\geq$ one episode of ON and myelitis and $\geq$ two of three supportive criteria: magnetic resonance imaging (MRI) evidence of a contiguous spinal cord lesion three or more vertebral segments in length, onset brain MRI non-diagnostic for MS or NMO-immunoglobulin $\mathrm{G}(\mathrm{IgG})$ seropositivity. ${ }^{6}$

The following data were gathered from the database and from medical charts in Düsseldorf and Innsbruck: sex, ethnicity, age at onset, symptoms at onset, course of the disease; defined as monophasic ADEM (one attack) or multiphasic ADEM (> one attack), duration of follow-up, number of ON attacks, bilateral ON, presence of residual vision loss, cerebral and spinal MRI data, occurrence of pleocytosis or oligoclonal bands (OCBs) in cerebrospinal fluid (CSF). NMO-IgG status was analyzed in serum by radioimmunoprecipitation assay (RIPA) as described elsewhere. ${ }^{7}$

\section{Analysis of MOG antibodies}

All serum samples were analyzed for the presence of antiMOG-IgG antibodies by an immunofluorescence assay using transiently transfected MOG expressing cells as previously described. ${ }^{3,8,9}$ Screening was performed at a 1:20 and 1:40 dilution by two clinically blinded investigators, and positive serum samples were further diluted to determine the titer levels of antibodies.

\section{Results}

\section{Patient detection and analysis of prevalence}

One hundred and thirty-two patients with inflammatory demyelinating disorders were included in the database between the years 2000 and 2010. Of these, 72 (54\%) had experienced at least one episode of ON. Of these patients, $54(75 \%)$ fulfilled the diagnostic criteria for MS. Of the remaining 18 patients, six fulfilled the criteria for NMO.

Seven patients had had at least one episode of ON but never had ADEM. Four were females, median age at first presentation was 10 years (range 4 to 14 years), median number of $\mathrm{ON}$ attacks was two (range one to three). In three patients residual vision loss was detected at last presentation. OCBs in CSF were negative in all patients and cell counts ranged between 1 and $12 / \mu$ l (median 1). Cranial MRI was normal in all patients and all were negative for NMO-IgG in serum. Six of these patients were investigated for anti-MOG antibodies in serum and two were found to be positive. Interestingly, those were the only two patients who had three ON attacks.

Five patients fulfilled all search criteria for ADEM followed by ON: $\geq$ one episode of ADEM and $\geq$ one episode with ON. One of these patients was excluded from the study because parental consent for the publication of the patient data could not be obtained.

After the search in our database, collaborating physicians at four university hospitals were asked for patients with $\geq$ one episode of ADEM and $\geq$ one episode with ON, and three more patients were included one from Innsbruck and two from Düsseldorf. The clinical and paraclinical characteristics of all patients are summarized in Table 1 and the clinical course in Figure 1. All patients but one were of European origin, five were female. Follow-up was between 21 months and 10 years (median six years).

\section{Presentation of ADEM}

The age at first presentation varied between 4 and 8 years (median 6 years). All patients were hospitalized in an acutely unwell state with suspected encephalitis. Clinical symptoms at the initial episode included fever $(n=5)$, headaches $(n=$ $6)$, meningeal signs $(n=2)$, lethargy $(n=7)$, recurrent vomiting $(n=3)$, seizures $(n=1)$, paraparesis $(n=1)$, tetraparesis $(n=1)$, ataxia $(n=1)$, bladder dysfunction $(n=1)$ and hallucinations $(n=1)$. Two patients (Pats 1 and 7$)$ had myelitis during an ADEM attack. Three patients showed multiphasic ADEM. Only in Pat 1 did the ADEM result in residual symptoms, a mild spastic paraparesis and bladder dysfunction. Six patients (Pats 1-3,5-7) received steroids for this treatment of ADEM, leading to an improvement of symptoms. 
Table I. Clinical and laboratory characterization of patients.

\begin{tabular}{|c|c|c|c|c|c|c|c|c|c|c|c|c|}
\hline \multirow[t]{2}{*}{ Patient } & \multirow[t]{2}{*}{ Sex } & \multirow[t]{2}{*}{ Ethnicity } & \multicolumn{6}{|c|}{ ADEM } & \multirow{2}{*}{ 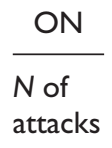 } & \multirow{2}{*}{$\begin{array}{l}\text { Residual } \\
\text { symptoms }\end{array}$} & \multirow{2}{*}{$\frac{\text { MOG-IgG }}{\text { Titer }}$} & \multirow[t]{2}{*}{ NMO-lgG } \\
\hline & & & $\begin{array}{l}\text { Age } \\
\text { (years) }^{\mathrm{a}}\end{array}$ & $\begin{array}{l}\text { Attacks } \\
\text { (n) }\end{array}$ & Symptoms & $\begin{array}{l}\text { CSF } \\
\text { pleocytosis }\end{array}$ & OCBs & $\begin{array}{l}\text { MRI typical } \\
\text { for ADEM }\end{array}$ & & & & \\
\hline I & $\mathrm{F}$ & e & 8 & 3 & $\begin{array}{l}\mathrm{Fe}, \mathrm{H}, \mathrm{L}, \mathrm{V}, \\
\mathrm{S}, \mathrm{P}\end{array}$ & + & - & + & 9 & + & $\begin{array}{l}I: 320 \\
I: 1280\end{array}$ & - \\
\hline 2 & $\mathrm{~F}$ & e & 4 & 2 & $\begin{array}{l}\mathrm{Fe}, \mathrm{H}, \mathrm{L}, \mathrm{V}, \\
\mathrm{Ha}\end{array}$ & + & - & + & 8 & + & $\mathrm{I}: 640$ & - \\
\hline 3 & $M$ & e & 5 & I & $\begin{array}{l}\mathrm{Fe}, \mathrm{H}, \mathrm{M} \\
\mathrm{L}, \mathrm{V}\end{array}$ & + & - & + & 2 & + & $\mathrm{I}: 1280$ & - \\
\hline 4 & $\mathrm{~F}$ & e & 6 & I & $\mathrm{Fe}, \mathrm{H}, \mathrm{M}, \mathrm{L}$ & + & - & nd & 2 & - & $\mathrm{I}: 1280$ & - \\
\hline 5 & $M$ & e & 7 & I & $H, L, A x$ & + & - & + & I & + & $\begin{array}{l}\mathrm{I}: 20480 \\
\mathrm{I}: 1280 \\
\mathrm{I}: 5 \mathrm{I} 20\end{array}$ & - \\
\hline 6 & $\mathrm{~F}$ & e & 5 & I & $\mathrm{H}, \mathrm{L}$ & - & - & + & 1 & - & $\begin{array}{l}I: 1280 \\
I: 2560\end{array}$ & - \\
\hline 7 & $\mathrm{~F}$ & A & 6 & 4 & Fe, L, T, B & + & - & + & I & - & $\begin{array}{l}I: 1280 \\
I: 160 \\
I: 1280\end{array}$ & - \\
\hline
\end{tabular}

ADEM: acute disseminated encephalomyelitis; ON: optic neuritis; MOG: myelin-oligodendrocyte glycoprotein; lgG: immunoglobulin G; NMO: neuromyelitis optica; CSF: cerebrospinal fluid; OCBs: oligoclonal bands; MRI: magnetic resonance imaging; a: at first attack; F: female; M: male; e: European; A:Asian; Fe: fever; H: headaches; M: meningeal signs; L: lethargy; V; vomiting; S: seizures; P: paraparesis; T: tetraparesis; Ha: hallucinations; Ax: ataxia; B: bladder dysfunction; nd: not done.

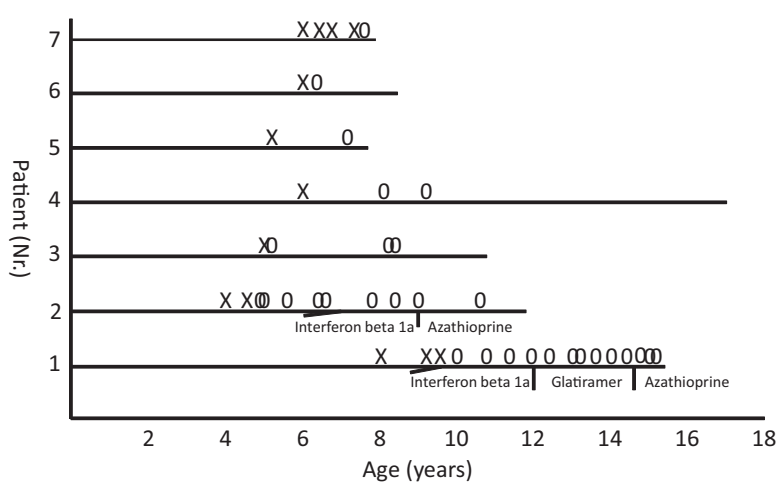

Figure I. Clinical course of patients with acute disseminated encephalomyelitis (ADEM) optic neuritis (ON). For the two treated patients, drugs are indicated. X:ADEM; 0: optic neuritis.

\section{Presentation of $\mathrm{ON}$}

The number of ON attacks varied between one and nine. The interval between ADEM and ON varied between three weeks and two years (Pat 1: three months; Pat 2: three weeks; Pat 3: five weeks; Pat 4: two years; Pat 5: 13 months; Pat 6: three months, Pat 7: six weeks). They were unilateral in six patients and both bilateral and unilateral in one (Pat 2). Visual-evoked potentials (VEP) studies were pathological in all patients during the course of ON. Four patients showed residual vision loss (visual acuity: Pat 1 measured repeatedly in between the ON attacks: right eye 1.0, left eye 0.8; Pat 2 measured six months following the last ON attack: right eye 0.8 , left eye 0.5 ; Pat 3 measured six months following the last $\mathrm{ON}$ attack: right eye 0.8 , left eye 1.0; Pat 5 measured four months following the ON attack: right eye 0.6 , left eye 0.9 ). All patients received steroids as a treatment for each of the ON attacks, resulting in an improvement in all. Two patients suspected of atypical pediatric MS were treated with immunomodulatory therapy including interferon beta and glatiramer acetate. In both patients the treatment did not seem to influence the frequency of ON episodes. Two patients received azathioprine.

\section{Laboratory studies}

In all patients but one, CSF studies at the time of ADEM showed a pleocytosis with a white cell count between 7 and $81 / \mu 1$. OCBs were negative in all. CSF was repeatedly analyzed in five of the patients during the later course of the disease and was consistently negative for OCBs (twice in Pats 1, 3, 4 and 5; four times in Pat 2). NMO-IgG status was not determined at the time of initial presentation but later on development of recurrent ON and was negative in all patients. Anti-MOG antibodies were determined during the initial ADEM attack in three patients (Pats 5-7) and were elevated, with titers ranging from 1:1280 to 1:20480 (Table 1). Anti-MOG antibodies during an acute episode of ON were elevated in all patients, with titers ranging from 1: 320 to 1:5120. Follow-up serum samples in four children revealed persistently high anti-MOG antibodies in the interval between and during episodes of $\mathrm{ON}$, with titers ranging from 1:160 to $1: 5120$. 
(a)

(b)
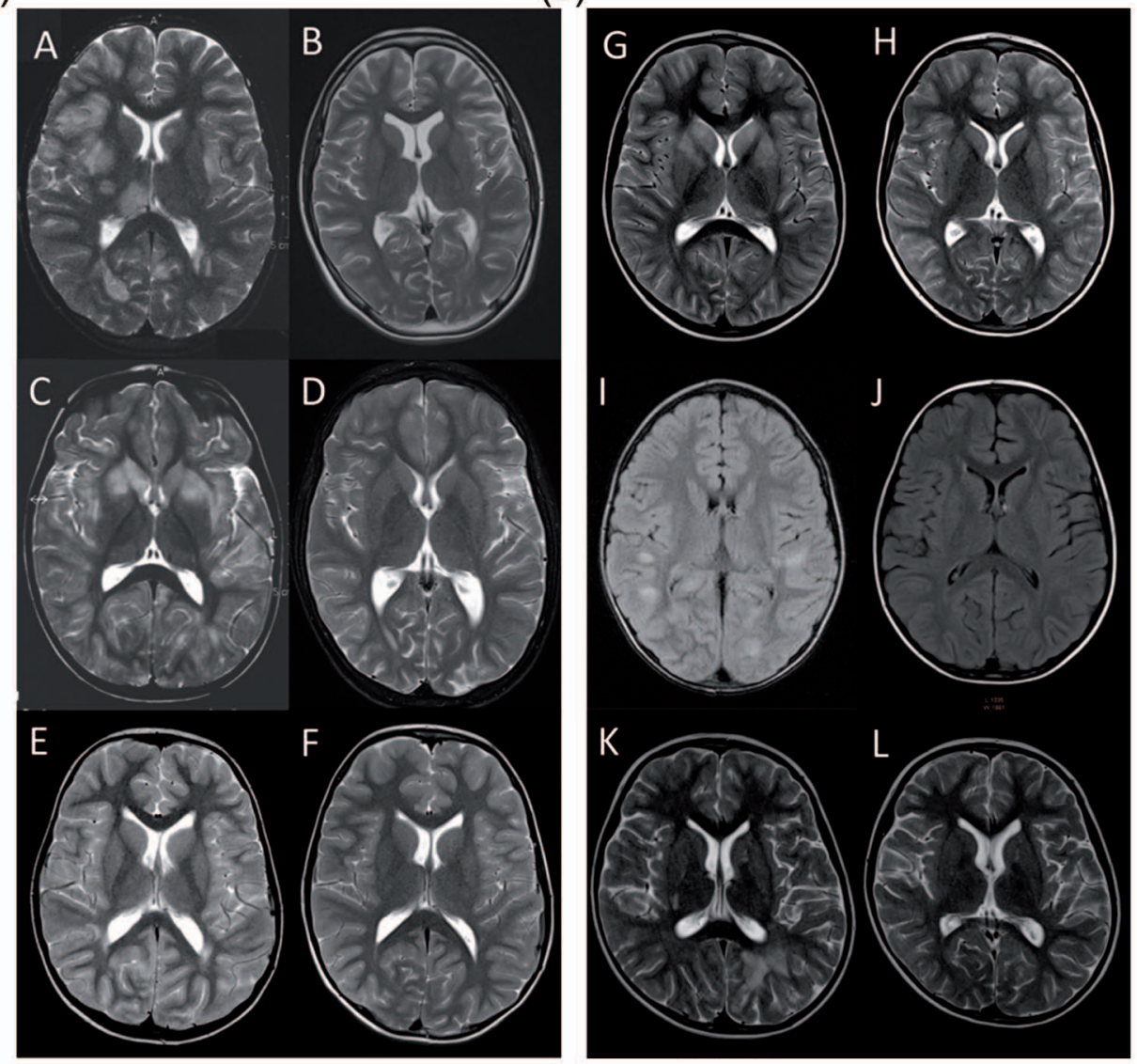

Figures $2 \mathbf{a}$ and $\mathbf{b}$. Cranial magnetic resonance imaging (MRI) of the patients. Patient I (A, B), Patient 2 (C, D) Patient 3 (E, F), Patient $5(\mathrm{G}, \mathrm{H})$, Patient $6(\mathrm{I}, \mathrm{J})$ and Patient $7(\mathrm{~K}, \mathrm{~L})$ at the time of first presentation with acute disseminated encephalomyelitis (ADEM), showing hyperintense lesions on $\mathrm{T} 2$ or fluid-attenuated inversion recovery (FLAIR)-weighted images affecting white and gray matter (A, $C, E, G, I, K)$ and during later episodes with optic neuritis $(O N)$, showing resolution of old lesions and no new lesions $(B, D, F, H, J, L)$.

\section{MRI studies}

Cranial MRI studies during the initial ADEM attack were performed in six patients and showed hyperintense T2 lesions involving both white and gray matter including the basal ganglia (Figure 2). There was no contrast enhancement. In one patient, Pat 4 , a computed tomography scan (CT) was performed at the initial attack but did not show alterations. Cranial MRI during subsequent ADEM attacks in Pats 1, 2 and 7 revealed new T2 lesions, again without contrast enhancement. Follow-up cranial MRI studies showed complete or almost complete resolution of the T2 lesions. Cranial MRI performed during all episodes of $\mathrm{ON}$ in all patients and on follow-up in six monthly intervals showed no new T2 lesions or contrast-enhancing lesions. Spinal MRI during the initial ADEM showed myelitis in Pats 1, 3 and 7.

\section{Discussion}

In the pediatric population the immune system leads to presentations of inflammatory demyelinating disorders that differ from those known in adults. It is not uncommon, for example, for patients with pediatric MS to initially present with ADEM. ${ }^{10,11}$ Nevertheless, some patients remain who cannot be classified under any of the defined disease entities, making their treatment and prognosis difficult. Here we describe a subgroup of such patients who shared clinical and paraclinical features and might therefore represent a recognizable distinct phenotype with common pathophysiology. All of these patients presented initially with one to four attacks of ADEM followed by one to nine attacks of ON. The prevalence of this phenotype was similar to NMO in our cohort of 132 patients with inflammatory demyelinating disorders. ADEM presentation included fever, headaches and lethargy in the majority. All but one patient displayed pleocytosis in CSF and all were negative for OCBs. MRI, performed in six patients during ADEM, showed typical T2 lesions that resolved completely or almost completely during the course of the disease. Repeated cranial MRI in all patients during the $\mathrm{ON}$ attacks and on follow-up did not reveal any new lesions. 
Distinction between the cohort of patients described in this article and pediatric MS is difficult and yet most important because of its therapeutic implications. All patients experienced at least two demyelinating events; however, the initial episodes were consistent with the diagnosis of ADEM and cannot therefore be considered as the first episodes of MS according to consensus criteria for pediatric MS. ${ }^{2}$ Additional aspects are also not supportive of pediatric MS in these patients. Firstly, CSF analyses were repeatedly negative for OCBs while in our cohort of pediatric MS patients $96 \%$ were positive. ${ }^{5}$ Furthermore, cranial MRIs showed no lesions typical for MS, no new T2 lesions on follow-up and almost complete resolution of old lesions (Figure 1).

In all patients anti-MOG antibodies were analyzed in serum because several studies have detected anti-MOGIgG antibodies primarily in serum of children with MS or ADEM. ${ }^{8}$ According to our observations (unpublished data) anti-MOG antibodies are only measurable in CSF in cases with high serum anti-MOG-IgG titers $(>1: 640)$. We therefore assume that anti-MOG IgG antibodies in the CSF are not produced intrathecally but derive from the blood periphery. However, Brilot et al. found an intrathecal IgG synthesis of anti-MOG antibodies in children with a clinically isolated syndrome (CIS) but not in children with ADEM. ${ }^{12}$

Interestingly, in all patients high titers for anti-MOG antibodies were detected at the initial presentation or at subsequent episodes. High titers of anti-MOG antibodies have been found in patients with ADEM, in pediatric patients with recurrent $\mathrm{ON}$ and recently in NMO patients negative for NMO-IgG. $3,9,12,13$ They are only rarely detected in monophasic $\mathrm{ON}$ and in patients with $\mathrm{ON}$ as a part of a CIS. In the patients from the Göttingen cohort of patients with inflammatory CNS disorders who presented with ON or recurrent $\mathrm{ON}$ alone, the only patients who had more than two ON episodes were found to be seropositive for antiMOG antibodies. The finding of high titers of anti-MOG antibodies in our study is therefore in concordance with previous studies on anti-MOG antibodies in pediatric patients. We hypothesize that persisting high anti-MOG antibodies are associated with a spectrum of recurrent demyelinating disorders including recurrent ADEM, recurrent $\mathrm{ON}$, recurrent $\mathrm{ADEM}$ followed by recurrent $\mathrm{ON}$ as described in this article and NMO-IgG negative NMO.

MOG-specific antibodies have been shown to mediate demyelination and exacerbate disease severity in vitro and several animal models of MS, suggesting that they potentially can contribute to lesion formation in children and adults with MS/ADEM who are positive for serum antiMOG antibodies. ${ }^{14,15}$ Recent data also indicate that these antibodies recognize the correct membrane topology and glycosylation pattern of MOG. ${ }^{16}$ Although these results indicate a pathogenic potential of anti-MOG antibodies, there is an ongoing controversy about their contribution to the disease process in humans.
Due to the relatively small number of patients, one has to be very careful about drawing conclusions regarding treatment. In all patients high-dose corticosteroids were given for ADEM and ON attacks and appeared to have a beneficial effect, resulting in at least a faster recovery. Two patients received immunomodulatory treatment (interferon beta 1a) for recurrent ON that did not seem to alter the frequency or severity of the attacks. In one patient azathioprine potentially reduced the number of $\mathrm{ON}$ attacks but in a second patient it did not.

Data on prognosis are also limited as only in four patients (Pats 1-4) did follow-up exceed five years. In Pats 1 and 2 the disease is clearly ongoing, in Pat 3 the attacks have a frequency too low to allow for any conclusions regarding the future course and in Pat 4 the disease seems to have stopped, indicating that there is a significant variety of disease activity. Presently the question as to whether this condition is self-limiting or a lifelong disorder can therefore not be answered. Residual symptoms, mainly mild vision loss, were present in four patients, especially in those with multiple ON attacks. The prognosis seems to be better than in NMO whereby within five years of disease onset, more than half of the patients suffer severe vision loss in at least one eye or will be unable to ambulate without assistance. ${ }^{17}$ Interestingly, in a recent study of NMO adults patients, those without NMO-IgG but with anti-MOG antibodies were found to have a more favorable outcome. ${ }^{18}$

In conclusion, we report on a cohort of pediatric patients with a demyelinating disorder characterized by recurrent or monophasic ADEM followed by ON. The prevalence was comparable to NMO in our pediatric patient cohort. Other hallmarks are negativity for OCBs in CSF and high titer for anti-MOG antibodies in serum. Reports of additional prospectively followed patients are needed before treatment recommendations or substantial predictions on prognosis can be made.

\section{Funding}

This research received no specific grant from any funding agency in the public, commercial, or not-for-profit sectors.

\section{Conflict of interest}

The authors declare no conflicts of interest.

\section{References}

1. Banwell B, Bar-Or A, Arnold DL, et al. Clinical, environmental, and genetic determinants of multiple sclerosis in children with acute demyelination: A prospective national cohort study. Lancet Neurol 2011; 10: 436-445.

2. Krupp LB, Banwell B and Tenembaum S. Consensus definitions proposed for pediatric multiple sclerosis and related disorders. Neurology 2007; 68: S7-S12.

3. Rostasy K, Mader S, Schanda K, et al. Anti-myelin oligodendrocyte glycoprotein antibodies in pediatric patients with optic neuritis. Arch Neurol 2012; 69: 752-756. 
4. Huppke P, Bluthner M, Bauer O, et al. Neuromyelitis optica and NMO-IgG in European pediatric patients. Neurology 2010; 75: 1740-1744.

5. Stark W, Huppke P and Gärtner J. Paediatric multiple sclerosis: The experience of the German Centre for Multiple Sclerosis in Childhood and Adolescence. J Neurol 2008; 255 (Suppl 6): 119-122.

6. Wingerchuk DM, Lennon VA, Pittock SJ, et al. Revised diagnostic criteria for neuromyelitis optica. Neurology 2006; 66: 1485-1489.

7. Paul F, Jarius S, Aktas O, et al. Antibody to aquaporin 4 in the diagnosis of neuromyelitis optica. PLoS Med 2007; 4: e133.

8. Di Pauli F, Mader S, Rostasy K, et al. Temporal dynamics of anti-MOG antibodies in CNS demyelinating diseases. Clin Immunol 2011; 138: 247-254.

9. Mader S, Gredler V, Schanda K, et al. Complement activating antibodies to myelin oligodendrocyte glycoprotein in neuromyelitis optica and related disorders. J Neuroinflammation 2011; 8: 184.

10. Mikaeloff Y, Adamsbaum C, Husson B, et al. MRI prognostic factors for relapse after acute CNS inflammatory demyelination in childhood. Brain 2004; 127: 1942-1947.

11. Neuteboom RF, Boon M, Catsman Berrevoets CE, et al. Prognostic factors after a first attack of inflammatory CNS demyelination in children. Neurology 2008; 71: 967-973.
12. Brilot F, Dale RC, Selter RC, et al. Antibodies to native myelin oligodendrocyte glycoprotein in children with inflammatory demyelinating central nervous system disease. Ann Neurol 2009; 66: 833-842.

13. Probstel AK, Dornmair K, Bittner R, et al. Antibodies to MOG are transient in childhood acute disseminated encephalomyelitis. Neurology 2011; 77: 580-588.

14. Kerlero de Rosbo N, Honegger P, Lassmann H, et al. Demyelination induced in aggregating brain cell cultures by a monoclonal antibody against myelin/oligodendrocyte glycoprotein. J Neurochem 1990; 55: 583-587.

15. Linington C, Bradl M, Lassmann H, et al. Augmentation of demyelination in rat acute allergic encephalomyelitis by circulating mouse monoclonal antibodies directed against a myelin/ oligodendrocyte glycoprotein. Am J Pathol 1988; 130: 443-454.

16. Marta CB, Oliver AR, Sweet RA, et al. Pathogenic myelin oligodendrocyte glycoprotein antibodies recognize glycosylated epitopes and perturb oligodendrocyte physiology. Proc Natl Acad Sci U S A 2005; 102: 13992-13997.

17. Collongues N, Marignier R, Zephir H, et al. Neuromyelitis optica in France: A multicenter study of 125 patients. Neurology 2010; 74: 736-742.

18. Kitley J, Woodhall M, Waters P, et al. Myelin-oligodendrocyte glycoprotein antibodies in adults with a neuromyelitis optica phenotype. Neurology 2012; 79: 1273-1277. 